\title{
Efeito de diferentes tratamentos do substrato dental na resistência adesiva e fixação de restaurações indiretas associada a adesivo auto-condicionante Effect of different treatments of the dental substrate on the ultimate bond strength of indirect restorations associated to a self-etching adhesive
}

\author{
Carolina Guimarães CASTRO \\ Aluna de graduação - Iniciação Científica - Faculdade de Odontologia - Universidade Federal de Uberlândia - UFU \\ - Uberlândia - MG - Brasil \\ Paulo César Freitas SANTOS FILHO \\ Mestrando - Programa de Pós-Graduação - Faculdade de Odontologia - Universidade Federal de Uberlândia - UFU \\ - Uberlândia - MG - Brasil \\ Denildo MAGALHÃES \\ Professor - Disciplina - Periodontia da Faculdade de Odontologia - Universidade Federal de Uberlândia - UFU \\ - Uberlândia - MG - Brasil
}

Carlos José SOARES

Professor - Disciplinas - Dentística e Materiais Odontológicos da Faculdade de Odontologia - Universidade Federal de Uberlândia - UFU - Uberlândia - MG - Brasil

\begin{abstract}
Resumo
Este trabalho avaliou a resistência adesiva ( $\mu$ TBS) na fixação de restaurações indiretas por meio de cimento resinoso e adesivo auto-condicionante sob influência de diferentes tratamentos prévios de esmalte e dentina. Trinta incisivos bovinos foram extraídos e tiveram suas raízes cortadas na junção cemento-esmalte. A superfície vestibular dos dentes foi lixada até exposição de dentina superficial, padronizando a formação de smear layer. As amostras foram divididas aleatoriamente em três grupos $(\mathrm{n}=10)$ : $\mathrm{G1}$ : aplicação do adesivo auto-condicionante One up Bond F (OB), seguindo as instruções do fabricante; G2:condicionamento com ácido fosfórico à 37\% em esmalte e dentina seguindo aplicação de

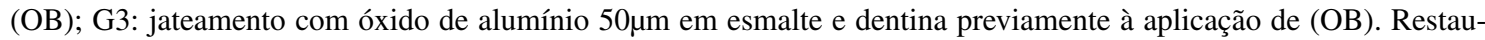
rações indiretas com resina composta foram fixadas usando cimento resinoso dual e as amostras foram cortadas com área adesiva de aproximadamente $1,0 \mathrm{~mm}^{2} \mathrm{em}$ três regiões: esmalte (E), dentina periférica (DP) e dentina central (DC). Foram submetidas a ensaio de microtração em máquina de ensaio mecânico em velocidade de $0,5 \mathrm{~mm} / \mathrm{min}$. Após fratura, as amostras foram analisadas em Microscopia Eletrônica de Varredura (MEV) para avaliação do padrão de fratura. Os valores de ( $\mu \mathrm{TBS}$ ) foram expressos em MPa e os dados foram analisados em ANOVA seguido por teste Tukey $(\mathrm{p}<0,05)$.

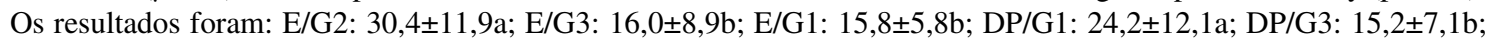

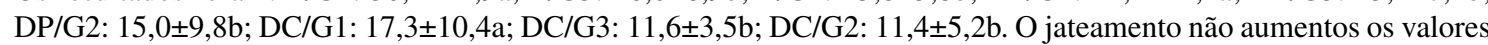
de $(\mu \mathrm{TBS})$. O prévio condicionamento ácido aumentou a resistência adesiva em esmalte e diminuiu em dentina.
\end{abstract}

\section{UNITERMOS}

Adesivos dentinários, ácidos fosfóricos; análise de variância; animal

\section{INTRODUÇÃo}

Os princípios clássicos da Odontologia Restauradora têm sido continuamente desafiados nas últimas duas décadas, tornando-se a tecnologia adesiva cada vez mais fundamental. Os materiais restauradores interagem com a dentina de várias formas, quer mecânica, quimicamente, ou de ambos os mecanismos ${ }^{22}$.
O conceito de adesão à dentina é baseado na premissa de que a este substrato caracteriza-se como composto biológico de mineral de apatita e fibras colágenas de alta resistência. Se a porção mineral for extraída por ácidos e, em seguida, substituída por monômeros resinosos, converte-se de composto biológico natural em composto terapêutico artificial ${ }^{4}$. Mais especificamente, o condicionamento ácido re- 
move detritos, mineral das áreas peri e intertubular expondo fibras colágenas que, quando corretamente tratadas, permitem que o monômero resinoso penetre por entre elas e, ao converter-se em polímero, ocorra a formação da camada híbrida ${ }^{23}$.

Uma freqüente manifestação clínica de pacientes submetidos a processos restauradores dentais é a sensibilidade pós-operatória ${ }^{2,21}$. Dentre os novos sistemas com número de passos operatórios reduzidos, os auto-condicionantes parecem ser menos sensíveis às variações de técnica e de substrato ${ }^{8}$. Os sistemas adesivos convencionais de frasco único proporcionam alta resistência de adesão, porém, possuem fatores que podem influenciar negativamente neste processo, como o risco de colapso das fibras colágenas durante a secagem da dentina após o condicionamento ácido $^{12-3,17}$. A grande vantagem dos sistemas auto-condicionantes reside no fato de que a desmineralização da superfície e infiltração dos monômeros resinosos ocorrem simultaneamente, eliminando a difícil tarefa de manter a umidade ideal de superfície, como ocorre com os sistemas em que esses passos são realizados separadamente.

Por outro lado, os sistemas auto-condicionantes ainda apresentam limitações em relação à interação com o esmalte devido à ausência da etapa do condicionamento ácido de esmalte, funcionando o próprio primer como condicionador. Isto origina padrões de condicionamento pouco profundos ${ }^{14}$, sugerindo menores valores de força de adesão ao esmalte ${ }^{11}$. Com isso diversos protocolos adicionais vem sendo postulados para otimizar a interação com este substrato, porém podem agir de forma adversa na união à dentina.

É pertinente, portanto, a análise da hipótese de que o condicionamento ácido e o jateamento prévios em esmalte e dentina, influenciem na resistência de adesão de adesivo auto-condicionante.

\section{MateriaIs e MÉtodos}

Foram selecionados trinta incisivos bovinos de animais adultos, recém extraídos e armazenados em solução aquosa tamponada de timol a $0,2 \%$. Os dentes foram limpos com curetas periodontais e submetidos à profilaxia com pedra pomes e água, sendo então, armazenados em água destilada sob refrigeração. Foram então, seccionados na junção amelo-cementária, com o auxílio de disco diamantado dupla face sob refrigeração, extraindo-se a porção central da coroa que foi fixada sobre placa acrílica com adesivo à base de cianoacrilato e godiva. A superfície vestibular da coroa foi desgastada com lixas de carbeto de silício (3M, St. Paul, MN, USA) de granulometria de 180, 320 e 600, respectivamente, até a exposição da dentina, padronizando a formação de smear layer. As amostras foram divididas aleatoriamente em três grupos $(n=10)$. Foram seguidos os seguintes protocolos de tratamento do substrato dental. No grupo G1, foi utilizado o sistema adesivo auto-condicionante One Up Bond F (Tokuyama, Tókio, Japão), seguindo as instruções do fabricante. No grupo G2, foi utilizado o sistema adesivo auto-condicionante One Up Bond F (Tokuyama) após prévio condicionamento com ácido fosfórico a 37\% (Scotchbond etching, 3M-ESPE, St. Paul, MN, USA), em esmalte e dentina, durante 15 segundos, lavagem pelo mesmo tempo e secagem com papel absorvente. No grupo G3, foi realizado jateamento prévio (Microjato, Bio Art, São Carlos, SP, Brasil) do substrato dental com óxido de alumínio $50 \mu$ (Bio Art, São Carlos, SP, Brasil) durante 10 segundos com a ponta do aparelho posicionada perpendicularmente a superfície a $1 \mathrm{~cm}$ da amostra e seguido o protocolo de G1. Os espécimes foram armazenados em umidade relativa a $37^{\circ} \mathrm{C}$ por 24 horas.

Foram confeccionadas trinta restaurações de resina composta TPH Spectrum (Dentsply, Petrópolis, RJ, Brasil) cor A3 de $11 \mathrm{~mm}$ de comprimento, por $7 \mathrm{~mm}$ de largura e $4 \mathrm{~mm}$ de espessura em matriz de polissulfeto (Permelastic, Kerr, USA) através de dois incrementos de $2,0 \mathrm{~mm}$ cada, e polimerizados por 40 segundos com unidade fotopolimerizadora XL 3000 com intensidade de luz de $500 \mathrm{~mW} / \mathrm{cm} 2$ (3M-ESPE, St. Paul, MN, USA). Essas restaurações foram póspolimerizadas em aparelho de nitrogênio - Nitroceram (Fotoceram, Catalão, GO, Brasil). Foi realizado o tratamento de superfície com jateamento de óxido de alumínio 50u e cimentação com cimento resinoso de dupla ativação RelyX ARC (3M-ESPE, St. Paul, MN, USA) fotoativado com aparelho fotopolimerizador XL 3000 com intensidade de luz de $500 \mathrm{~mW} / \mathrm{cm} 2$ (3MESPE, St. Paul, MN, USA) por 40 segundos em cada face da restauração.

O conjunto foi posicionado em micrótomo de tecido duro (ISOMET 1000, Buehler, UK Ltd.), e então seccionado com disco de diamante, (Extec 4"x 0,12 x 0,12, Enfield, CT, USA) em velocidade de corte calibrada em 400 RPM no sentido mésio-distal e inciso-cervical, resultando em cerca de dois palitos em dentina central, dois palitos em dentina periférica e 2 em esmalte, totalizando seis palitos para cada amostra com área adesiva de $\pm 1 \mathrm{~mm}^{2}$. Os espécimes foram armazenados em umidade relativa a $37^{\circ} \mathrm{C}$ por 
24 horas. As amostras foram fixadas ao dispositivo de microtração com adesivo a base de cianoacrilato $(\mathrm{Su}-$ per Bonder, Loctite, SP, Brasil) e acelerador de presa (Loctite, PK280, SP, Brasil) e submetidas a ensaio mecânico em máquina (EMIC DL 2000, São José dos Pinhais, Brasil) a uma velocidade de $0,5 \mathrm{~mm} /$ minuto. Após a fratura, foi capturado o valor de carga máxima em Kgf. Com auxílio de paquímetro digital (Starret, Itu, SP, Brasil) foi mensurada a área da superfície fratura. A carga máxima foi relacionada com área adesiva, definindo-se, assim, a resistência adesiva em MPa. Os dados foram então submetidos a análise de variância fatorial $3 \times 3$ e teste de Tukey em nível de significância de $5 \%$.

Duas amostras de cada grupo foram fixadas a um stub metálico. Esse conjunto foi metalizado, com aplicação de camada de ouro/paládio sobre sua superfície, num metalizador. As superfícies foram analisadas em Microscópio Eletrônico de Varredura de pressão variada, em alto vácuo (LEO 435 VP, Carl Zeiss, Alemanha) para definição qualitativa do padrão de fratura. Os tipos de fratura foram classificados em fratura coesiva (envolvendo fratura do substrato dental, camada híbrida, cimento ou restauração), adesiva (aparente descolamento do sistema adesivo e cimento resinoso do substrato dentinário) e mista (associação de fratura coesiva e adesiva entre camada adesiva e dentina).

\section{Resultados}

Os dados apresentaram distribuição normal e homogênea. A análise de variância revelou que existiram diferenças estatisticamente significantes na resistência adesiva entre os diferentes tratamentos prévios do substrato, como mostra a Tabela 1 onde se encontram as médias dos valores analisados pelo teste Tukey. Em esmalte, o maior valor foi encontrado com associação do condicionamento ácido prévio (30,4 MPa), cuja observação em MEV mostrou fratura coesiva na restauração (Figuras 1 e 2).

\section{Tabela 1 - Resistência adesiva (Valores indicados por letras diferentes têm significados estatistica- mente diferentes, $p<0,05 ; n=10$ por grupo)}

\begin{tabular}{l|l|l}
\hline Grupo & Substrato dental & Valor (MPa) (DV) \\
\hline G1 & & $15.8(5.8)^{\mathrm{b}}$ \\
G2 & Esmalte & $30.4(11.9)^{\mathrm{a}}$ \\
G3 & $16.0(8.9)^{\mathrm{b}}$ \\
& & $24.2(12.1)^{\mathrm{a}}$ \\
G1 & Dentina periférica & $15.0(9.8)^{\mathrm{b}}$ \\
G2 & & $15.2(7.1)^{\mathrm{b}}$ \\
G3 & & $17.3(10.4)^{\mathrm{a}}$ \\
G1 & & $11.4(5.2)^{\mathrm{b}}$ \\
G2 & Dentina central & $11.6(3.5)^{\mathrm{b}}$ \\
G3 & & \\
\hline
\end{tabular}



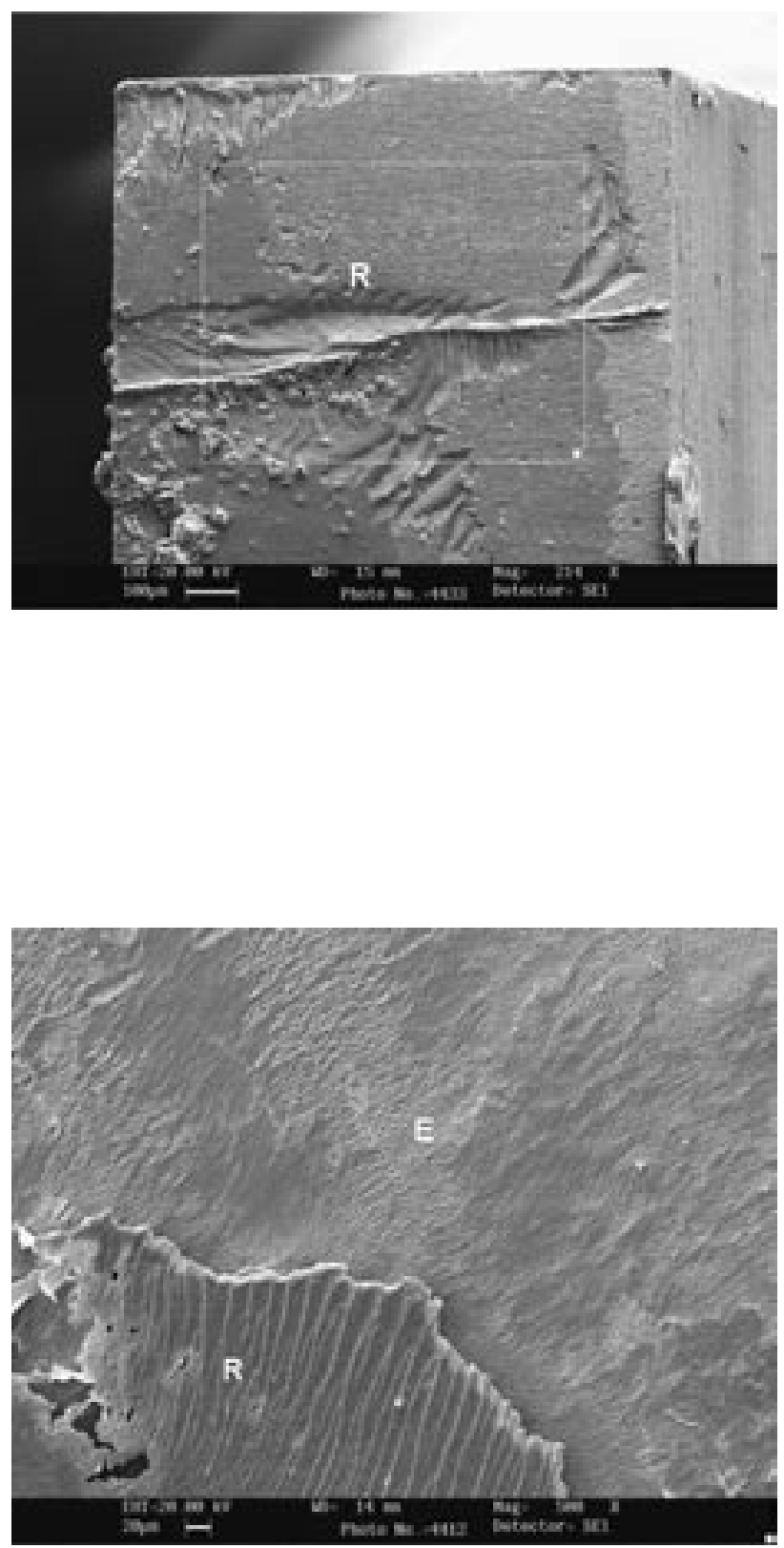

Nas duas regiões da dentina, os maiores valores foram apresentados pelo grupo onde foi realizado apenas aplicação do adesivo auto-condicionante. A análise de imagens em MEV demonstrou distribuição parcial equivalente entre fraturas coesivas entre dentina e adesivo e adesivas entre camada adesiva e cimento
FIGURA 1 - Fratura em esmalte de amostra que recebeu prévio condicionamento ácido - Ampliação original 214x ( $\mathrm{R}$ - restauração)

Figura 2 - Fratura em esmalte de amostra tratada apenas com adesivo auto condicionante - Ampliação original 500x ( $\mathrm{R}$ - restauração; E - esmalte)

resinoso (Figura 3). Quando o condicionamento ácido foi associado, o modo de fratura predominante foi entre o substrato dentinário e o sistema adesivo (Figura 4). Os espécimes que receberam prévio jateamento com óxido de alumínio mostraram, principalmente, fraturas mistas (Figura 5). 
EFEITO DE DIFERENTES TRATAMENTOS DO SUBSTRATO DENTAL NA RESISTÊNCIA ADESIVA E FIXAÇÃO DE RESTAURAÇÕES INDIRETAS ASSOCIADA A ADESIVO AUTO-CONDICIONANTE
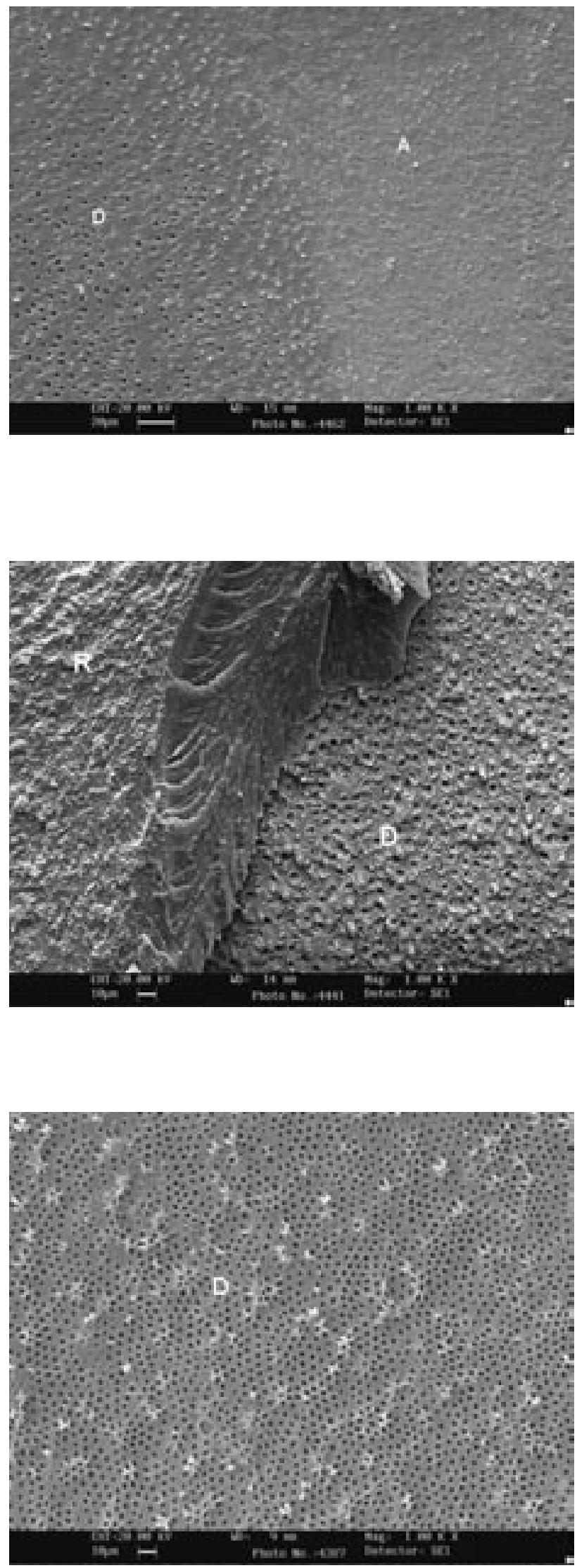

FIGURA 3 - Fratura em dentina de espécime que recebeu aplicação do adesivo seguindo as instruções do fabricante - Ampliação original 1000x (D - dentina; A - adesivo)

FIGURA 4 - Fratura em dentina de amostra que recebeu prévio condicionamento ácido - Ampliação original 1000x ( $\mathrm{R}$ - restauração; D - dentina)

FIGURA 5 - Fratura em dentina em amostra que recebeu jateamento com óxido de alumínio previamente a aplicação do sistema adesivo - Ampliação original 1000x (D - dentina). 


\section{Discussão}

Diante da evolução dos materiais odontológicos e principalmente da odontologia preventiva, os dentes humanos vêm sendo atualmente substituídos por dentes bovinos em estudos que envolvem resistência adesiva tanto em esmalte quanto em dentina ${ }^{16}$. As propriedades específicas da dentina, estrutura tubular e umidade intrínseca, fazem da adesão à dentina um procedimento mais criterioso que a adesão ao esmalte $^{6,20}$. $\mathrm{O}$ condicionamento ácido em esmalte transforma sua superfície lisa e suave em superfície acentuadamente irregular, permitindo que, diante da aplicação do material resinoso, ocorra a infiltração do monômero nas irregularidades produzindo uma interação micromecânica, estabelecendo a adesão ${ }^{3}$. $\mathrm{O}$ condicionamento produzido pelo One Up Bond

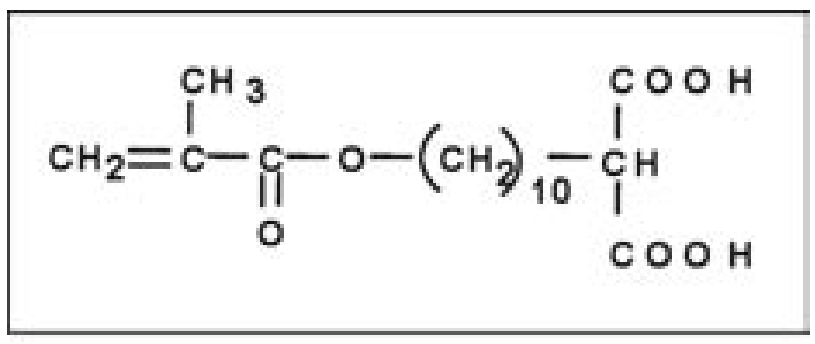

Em dentina, a associação do ácido fosfórico diminuiu os valores de resistência adesiva, os quais podem ser explicados por duas hipóteses: primeira, pelo fato de já existir concomitante condicionamento na aplicação do sistema adesivo One Up Bond F, resultando em duplo condicionamento, e a segunda razão seria a capacidade do ácido fosfórico de promover desnaturação protéica das fibras colágenas pela característica de ácido forte. Este acontecimento tem influência negativa no componente orgânico da dentina, o qual consiste em $20 \%$ do substrato dentinário ${ }^{15}$. Quando aplicado apenas o adesivo auto-condicionante, a maioria das fraturas ocorreu entre a camada adesiva e o cimento resinoso, em ambas as regiões, mostrando dessa forma, boa interação entre o substrato dentinário e o adesivo One Up Bond F. O que pode ser justificado também pela possível inibição de polimerização do cimento resinoso pelo monômero ácido remanescente na superfície.

Considerando as variações naturais do substrato dentinário era de se esperar que a contribuição da ca-
$\mathrm{F}$ é proveniente do componente ácido metacrilato. Diversos relatos demonstraram que sistemas adesivos auto-condicionantes apresentam mínima ou nenhuma penetração do adesivo na superfície do esmalte ${ }^{9,14} \mathrm{e}$, diante da associação de condicionamento ácido prévio com ácido fosfórico criam maior penetração de tags resinosos $^{9,14}$. De acordo com os resultados obtidos nesse estudo, confirma-se a influência de etapa de condicionamento com ácido fosfórico a $37 \%$ no aumento da resistência adesiva de união ao esmalte. A Figura 1 exemplifica a interface esmalte-resina das amostras que receberam prévio condicionamento ácido, onde pode-se observar fratura da restauração, demonstrando alta adesão esmalte/adesivo promovida pelo ácido fosfórico, o qual tem a capacidade de promover uma superfície mais irregular no esmalte que o MAC-10 (Figura 6).
FIGURA 6 - Fórmula do 10-ácido metacriloiloxidecametileno malônico (MAC-10).

mada híbrida e dos "tags" de resina também variem de acordo com a região da dentina. A dentina superficial apresenta poucos túbulos (cerca de $1 \%$ da área) e é composta na sua maior parte por dentina intertubular, favorecendo, portanto, a formação da camada híbrida ${ }^{7}$. Diversos estudos têm investigado a relação entre a microestrutura dentinária e suas propriedades mecânicas $^{5,10,18}$. Eles têm concluído que essas diferenças refletem em profundos efeitos na resistência adesiva ${ }^{4}$. Portanto, para o alcance de resultados mais fiéis, é fundamental a padronização de regiões na dentina na obtenção de amostras que serão submetidas a ensaio de microtração.

Relacionado ao jateamento prévio com óxido de alumínio, em esmalte não resultou em valores estatisticamente significantes quando comparados com o grupo controle e diminuiu a resistência adesiva em relação ao grupo 2. Contudo, outros estudos mostram que o uso isolado do jateamento em esmalte não é aconselhável para aplicação clínica ${ }^{1,19}$. Em dentina, o jato de óxido de alumínio resultou em valores similares na resistência 
adesiva quando comparados ao prévio condicionamento ácido. O que demonstra a ineficiência destes procedimentos para prévio tratamento do substrato dentinário que receberá adesivo auto condicionante.

Este estudo apresenta aspectos limitantes que podem ser explorados em novos experimentos que envolvam a simulação de envelhecimento das amostras, quer seja pelo armazenamento em meio de degradação da camada híbrida, quer seja pela simulação mecânica e térmica sobre as amostras. Outro aspecto que certamente cabe novas investigações é o emprego de maior número de materiais adesivos com diferentes composições, quer sejam adesivos auto-condicionantes convencionais ou principalmente outras formulações de adesivos simplificados. Outro aspecto que ainda suscita a formulação de novas pesquisas é a análise do efeito do tratamento prévio com condicionamento com ácido fosfórico a $37 \%$ isoladamente em esmalte, pois parece ser difícil a delimitação da junção amelodentinária e com isso pode haver efeito indesejável sobre a dentina periférica pela extrapolação da área de aplicação do ácido, com isso é pertinente a investigação de outros tratamentos prévios que possam ser aplicados simultaneamente sobre os dois substratos, esmalte e dentina, permitindo aumento da intensidade e estabilidade da união adesiva.

\section{Conclusão}

De acordo com a metodologia aplicada e a análise dos dados obtidos, pode concluir que:

- O condicionamento com ácido fosfórico aumentou a resistência adesiva em esmalte, e, por outro lado, diminui em dentina;

- Ojateamento prévio não potencializou a adesão, nem em esmalte nem em dentina.

\section{Agradecimentos}

Os autores estão agradecidos ao Prof. EW Kitajima (NAP/MEPA-ESALQ/USP) pelo suporte técnico na realização das imagens em microscópio eletrônico de varredura, e a UNESP - Universidade Estadual Paulista - Araraquara pela assistência no uso da máquina cortadeira.

\begin{abstract}
This study evaluated the microtensile bond strength ( $\mu T B S)$ of indirect restorations fixed with self-etching adhesive under influence of previous treatments on substrate dental. Thirty bovine incisors were seccioned at the cemento-enamel junction and were grounded as to get a flat superficial dentin. They were divided into 3 groups (n=10): G1-control; G2-enamel and dentin 37\% phosphoric acid etching; G3-enamel and dentin 50 4 m aluminum oxide sandblasting. Indirect composite restorations were fixed using luting resin cement. Samples were sectioned with approximately $1.0 \mathrm{~mm}^{2}$ adhesive area in three regions: enamel $(E)$, peripheral dentin $(D P)$, central dentin $(D C)$. They were tested at $0.5 \mathrm{~mm} / \mathrm{min}$ in a microtensile testing machine and examined under SEM. Data were analyzed by ANOVA and Tukey test $(p<0.05)$. The results were (MPa): $E / G 2: 30,4 \pm 11,9 a ; E / G 3: 16,0 \pm 8,9 b ; E / G 1: 15,8 \pm 5,8 b ; D P / G 1: 24,2 \pm 12,1 a ; D P / G 3: 15,2 \pm 7,1 b ; D P / G 2$ : $15,0 \pm 9,8 b ; D C / G 1: 17,3 \pm 10,4 a ; D C / G 3: 11,6 \pm 3,5 b ; D C / G 2: 11,4 \pm 5,2 b$. The sandblasting didn't increase $\mu T B S$. The previous phosphoric acid etching increased $\mu T B S$ on enamel and decreased the dentin bond strength.
\end{abstract}

\title{
UNITERMS
}

Dentin-bonding agents; phoshoric acid; analysis of variance 


\section{ReferênCiAs}

1. Abu Alhaija ES, Al-Wahadni AM. Evaluation of shear bond strength with different enamel pre-treatments. Eur J Orthod. 2004;26(2):17984.

2. Akpata ES, Sadiq W. Post-operative sensitivity in glass-ionomer versus adhesive resin-lined posterior composites. Am J Dent 2001;14(1):348 .

3. Asmussen E; Munksgaard EC. Bonding of restorative materials to dentine: status of dentine adhesives and impact on cavity desing and filling techniques. Int Dent J. 1988;38(2):97-104.

4. Carvalho RM. Adesivos dentinários. Fundamentos para aplicação clínica. Rev Dent Rest 1998;1(2):62-69.

5. Carvalho RM, Fernandes CAO, Villanueva R, Wang L, Pashley DH. Tensile strength of human dentin as a function of tubule orientation and density. J Adhes Dent. 2001;3(4):309-14.

6. Frankenberger R, Krämer N, Petschelt A. Fatigue behavior of different dentin adhesives. Clin Oral Invest. 1999;3(1):11-7.

7. Garberoglio R, Brännström M. Scanning electron microscopic investigation of human dentinal tubules. Arch Oral Biol. 1976;21(6):35562.

8. Giannini M, Carvalho RM, Martins LR, Dias CT, Pashley DH. The influence of tubule density and area of solid dentin on bond strength of two adhesive systems to dentin. J Adhes Dent. 2001;3(4):315-24.

9. Gordan VV, Vargas MA, Denehy GE. Interfacial ultraestructure of the resin-enamel region of three adhesive systems. Am J Dent. 1998;11(1):13-6.

10. Konishi N, Watanabe LG, Hilton JF, Marshall GW, Marshall SJ, Staninec M. Dentin shear strength: effect of distance from the pulp. Dent Mater. 2002;18(7):516-20.

11. Osborne JW; Summit JB. Mechanical properties and clinical perfomance of a gallium restorative material. Oper Dent. 1995;20(6):2415 .

12. Pashley DH; Ciucchi B; Sano H; Horner JA. Permeability of dentin to adhesive agents. Quintessence Int. 1993 Sept.;24(9):618-31.

13. Paul SJ; Welter DA; Ghazi M; Pashley D. Nanoleakage at the dentin adhesive interface vs microtensile bond strength. Oper Dent. 1999 May./June.;24(3):181-8.

14. Perdigao J, Lopes L, Lambrechts P, Leitao J, Van Meerbeek B, Vanherle G. Effects of a self-etching primer on enamel shear bond strengths and SEM morphology. Am J Dent. 1997;10(3):141-6.

15. Provenza DC. Fundamentals of oral histology and embryology. 2nd ed. Philadelphia: Lea \& Febiger; 1988. p.147-80.
16. Reis AF, Giannini M, Kavaguchi A, Soares CJ, Line SRP. Comparison of microtensile bond strength to enamel and dentin of human, bovine, and porcine teeth. J Adhes Dent. 2004;6(2):117-21.

17. Sano H; Takatsu T; Ciucchi B; Horner JA; Matthews WG; Pashley DH. Nanoleakage: leakage within the hybrid layer. Oper Dent. 1995;20(1):18-25

18. Staninec M, Marshall GW, Hilton JF, Pashley DH, Gansky SA, Marshall SJ, Kinney JH. Ultimate tensile strength of dentin: Evidence for a damage mechanics approach to dentin failure. J Biomed Mater Res. 2002;63(3):342-5.

19. Tão L, Xu X, Cui ZX. The effect of air abrasion on enamel composite resin adhesive strength. Shanghai Kou Qiang Yi Xue 2001;10(3):2312.

20. Tay FR, Gwinnett JA, Wei SH. Micromorphological spectrum of acid conditioned dentin following the application of water-based adhesive. Dent Mater. 1998;14(5):329-38.

21. Unemori M, Matsuya Y, Akashi A, Goto Y, Akamine A. Composite resin restoration and postoperative sensitivity: clinical follow-up in an undergraduate program. J Dent. 2001;29(1):7-13.

22. Van Meerbeek B; Perdigão J; Lambrechts P; Vanherle G. The clinical perfomance of dentin adhesives. J Dent. 1998;26(1):1-20.

23. Watanabe A, Nakabayashi N. SEM observation of treated dentin surface.Tokyo Ika Shika Daigaku Iyo Kizai Kenkyusho Hokoku. 1989;23:121-7.

Recebido em: 10/10/05 Aprovado em: 25/05/06

Carlos José Soares Faculdade de Odontologia Universidade Federal de Uberlândia Área de Dentística e Materiais Odontológicos Av. Pará, n 1720 - Campus Umuarama

Bloco 2B - Sala 2B-24 carlosjsoares@umuarama.ufu.br 38405-902 - Uberlândia - MG - Brasil

Telefone -34 -32182255 Fax -34 - 32182279 